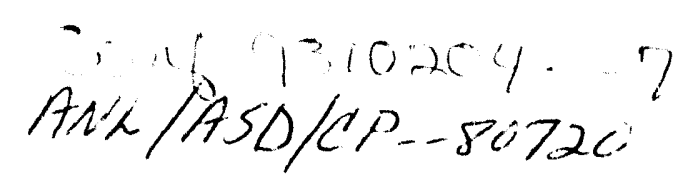

\title{
ON THE USE OF THE AUTOCORRELATION AND COVARIANCE METHODS FOR FEEDFORWARD CONTROL OF TRANSVERSE ANGLE AND POSITION JITTER IN LINEAR PARTICLE BEAM ACCELERATORS*
}

Dean S. Barr

Advanced Photon Source, Argonne National Laboratory, 9700 S. Cass Ave., Argonne, IL 60439

\section{ABSTRACT}

It is desired to design a predictive feedforward transverse jitter control system to control both angle and position jitter in pulsed linear accelerators. Such a system will increase the accuracy and bandwidth of correction over that of currently available feedback correction systems. Intrapulse correction is performed. An offline process actually "learns" the properties of the jitter, and uses these properties to apply correction to the beam. The correction weights calculated offline are downloaded to a real-time analog correction system between macropulses. Jitter data were taken at the Los Alamos National Laboratory (LANL) Ground Test Accelerator (GTA) telescope experiment at Argonne National Laboratory (ANL). The experiment consisted of the LANL telescope connected to the ANL ZGS proton source and linac. A simulation of the correction system using this data was shown to decrease the average rms jitter by a factor of two over that of a comparable standard feedback correction system. The system also improved the correction bandwidth.

\section{INTRODUCTION}

Figure 1 shows the standard setup for a feedforward transverse jitter control system. Note that one pickup \#1 and two kickers are needed to correct beam position jitter, while two pickup \#1's and one kicker are needed to correct beam trajectory-angle jitter.

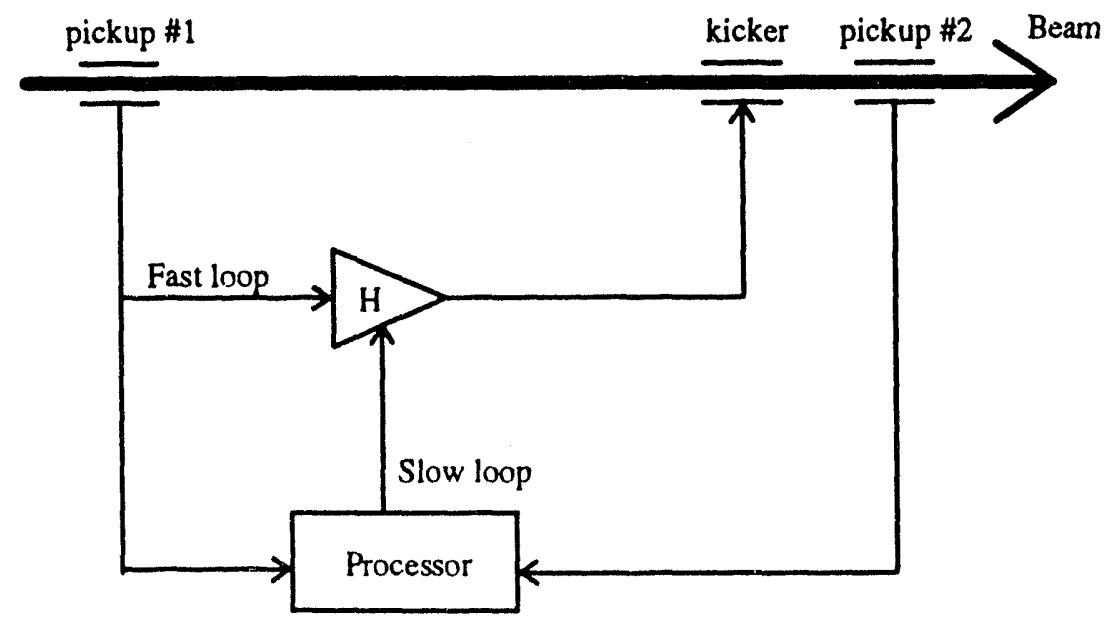

Figure 1. Feedforward Transverse Jitter Control System

It is assumed that the beam is fast enough to beat the correction signal (through the fast loop) to the kicker. The fast loop will be completely analog for maximum speed. $\mathrm{H}$ will take the form of an analog transverse filter with digitally adjustable gains. Such devices are commercially available. Figure 2 shows a typical transversal filter.

The slow loop will use digitized inputs and process the values for $\mathrm{H}$ in an offline computer. The updated values for $\mathrm{H}$ will be downloaded to the filter between macropulses. These values will be

\footnotetext{
* Work supported by the University of California under contract no. W-7405-ENG-36.
} 
chosen so that the output of the transversal filter, $\varphi$, will best predict the beam position at the kicker at some point in the future. The feasibility of this scheme depends on the degree of determinism (as opposed to stochasticity) of the beam jitter data. It will be shown that for at least one set of data (from an actual accelerator) there exist enough deterministic frequency components to both increase the accuracy of correction and extend the bandwidth of correction over that of a standard negative feedback correction system.

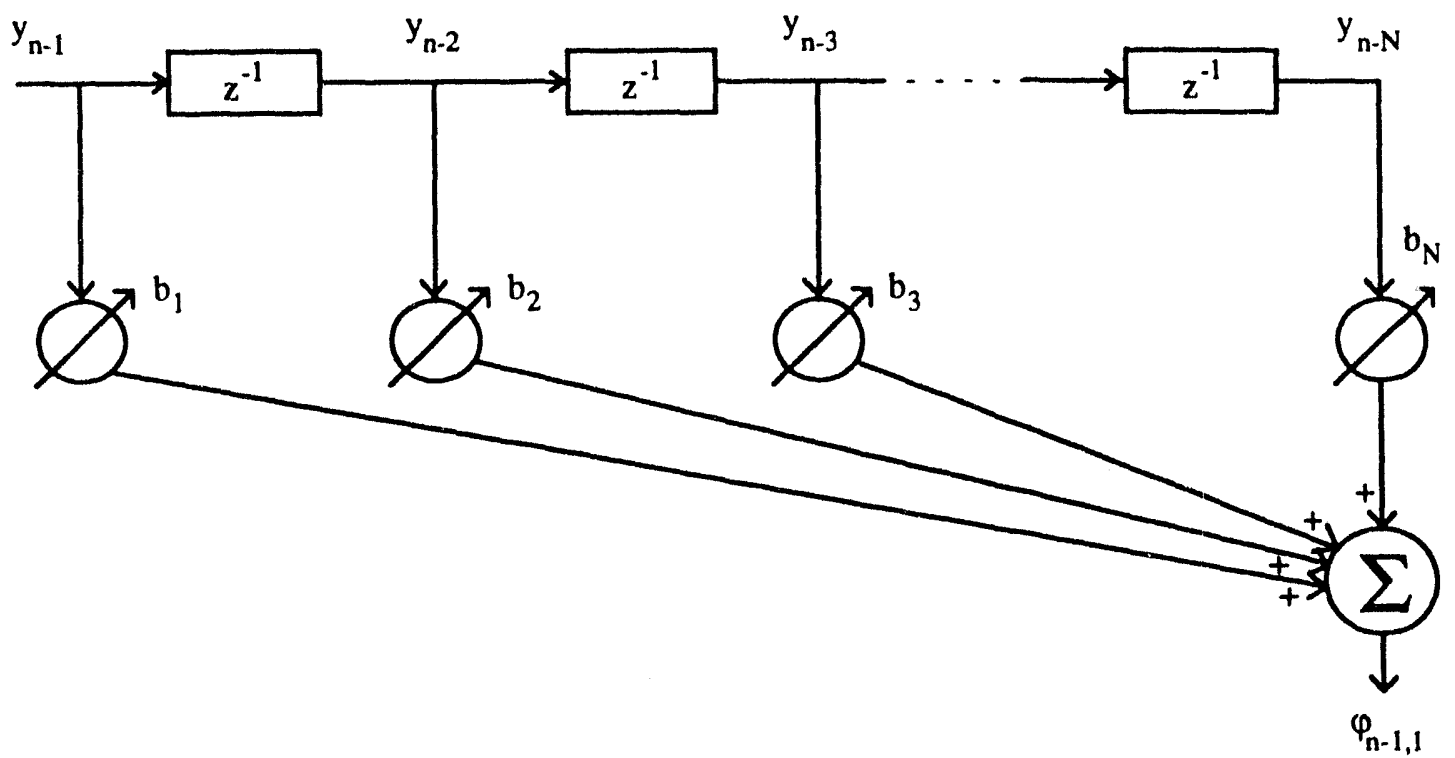

Figure 2. Transversal Prediction Filter $(v=1)$

\section{PREDICTION THEORY}

The most general form of a linear filter is found in Eq. (1)

$$
y_{k}=\sum_{k=0}^{M} a_{k} x_{n-k}+\sum_{j=1}^{N} b_{j} y_{n-j}
$$

where $x_{n}$ is the filter input and $y_{n}$ is the filter output. Taking the $z$-transform of Eq. (1) and rearranging gives

$$
H(z)=\frac{Y(z)}{X(z)}=\frac{\sum_{k=0}^{M} a_{k} z^{-k}}{1-\sum_{j=1}^{N} b_{j} z^{-j}} .
$$

If $\mathrm{M}=0$, this becomes 


$$
H(z)=\frac{a_{0}}{1-\sum_{j=1}^{N} b_{j} z^{-j}} .
$$

This is known as a recursive or infinite impulse response (IIR) filter. It is also called an autoregressive (AR) model, an all-pole model, or a maximum entropy method (MEM). Since it has poles, this filter has the possibility of becoming unstable. Define a new filter $\mathrm{A}(\mathrm{z})$ as the denominator of Eq. (3).

$$
A(z)=1-\sum_{j=1}^{N} b_{j} z^{-j}
$$

The zeroes of $A(z)$ are the poles of $H(z)$ and must lie inside the unit circle $(z<1)$ in order for $l_{1}(z)$ to be stable. If all the poles and zeroes of $A(z)$ lie inside the unit circle, $A(z)$ is a minimum jhase filter. The inverse of a minimum phase filter is always causal and stable. If $\mathrm{A}(\mathrm{z})$ has any zeroes outside the unit circle, $\mathrm{H}(z)$ will have at least one pole outside the unit circle and will be unstable. In this case $A(z)$ must be made minimum phase. This is done by reflecting inside the unit circle, any zeroes of $A(z)$ which lie outside the unit circle. This is done as follows. If $z_{\text {outside }}$ is the zero outside the unit ciricis, set $z_{\text {inside }}$ to

$$
z_{\text {inside }}=\frac{1}{z_{\text {outside }}^{*}}
$$

where * denotes the complex conjugate. This substitution preserves the magnitude of $\mathrm{H}(\mathrm{z})$ and only affects the phase. This is usually not a problem. This substitution is done after the coefficients have been determined. The stability is checked and Eq. (5) is applied if necessary.

The prediction equation can now be given by using $M=0$ in Eq. (1)

$$
\varphi_{n-v, v}=a_{0} x_{n}+\sum_{j=v}^{N} b_{j} y_{n-j}
$$

where $\varphi_{n-v, v}$ is the prediction for $y_{n}$ based on values of $y$ up to and including $y_{n-v}$. The variable $v$ is the number of points into the future into which the prediction is being made. Figure 2 shows a prediction filter with a value of $v=1$ (prediction one point into the future). The $a_{0} x_{n}$ term is the error between the prediction and the actual value and is not used as part of the prediction.

Referring to Figure 1, the values of $y$ are taken from pickup \#1. It is assumed that any jitter incurred between pickup \#1 and the kicker is completely deterministic and can be removed without error by information gained at pickup \#2. Thus, since the beam lattice is known in this region and any extra jitter is I lown, any predicted value of y at pickup \#1 can be translated to a value for correction at the kicker. Thus it is only necessary to develop a method for determining the b's in Eq. (6) in order to perform predictive correction. 
From Eq. (6), the prediction is now

$$
\varphi_{n-v, v}=\sum_{j=v}^{N} b_{j} y_{n-j}
$$

This is the prediction for $x_{n}$. The error in the prediction is

$$
\varepsilon_{n, v}=x_{n}-\varphi_{n-v, v}=x_{n}-\sum_{j=v}^{N} b_{j} x_{n-j} .
$$

It is desired to choose the $b_{j}$ 's in order to minimize the mean square error. The nature of the process $x_{n}$ will be dealt with in two cases: the deterministic and the random process case.

For the first case, assume that $x_{n}$ represents a deterministic signal. Compute the total squared error, $\mathrm{E}_{\mathrm{v}}$,

$$
E_{v}=\sum_{n} \varepsilon_{n, v}^{2}=x_{n}-\varphi_{n-v, v}=\sum_{n}\left(x_{n}-\sum_{j=v}^{N} b_{j} x_{n-j}\right)^{2} .
$$

The range of the summation for $n$ will be determined later. Now minimize $E_{v}$ by setting

$$
\frac{\partial E_{v}}{\partial b_{i}}=0, \quad v \leq i \leq N
$$

Expanding Eq. (9),

$$
\begin{aligned}
& E_{v}=\ldots+\left(x_{1}-\sum_{j=v}^{N} b_{j} x_{1-j}\right)^{2}+\left(x_{2}-\sum_{j=v}^{N} b_{j} x_{2-j}\right)^{2}+\ldots \\
& \frac{\partial E_{v}}{\partial b_{i}}=0=\ldots+2 x_{1-i}\left(x_{1}-\sum_{j=v}^{N} b_{j} x_{1-j}\right)+2 x_{2-i}\left(x_{2}-\sum_{j=v}^{N} b_{j} x_{2-j}\right)+\ldots \\
& \text { for } v \leq i \leq N \\
& \ldots+\left(x_{1} x_{1-i}-\sum_{j=v}^{N} b_{j} x_{1-j} x_{1-i}\right)+\left(x_{2} x_{2-i}-\sum_{j=v}^{N} b_{j} x_{2-j} x_{2-i}\right)+\ldots=0 \\
& \text { for } v \leq i \leq N \\
& \sum_{n}\left(-\sum_{j=v}^{N} b_{j} x_{n-j} x_{n-i}\right)=-\sum_{n} x_{n} x_{n-i} \quad \text { for } v \leq i \leq N
\end{aligned}
$$




$$
\sum_{j=v}^{N} b_{j} \sum_{n} x_{n-j} x_{n-i}=\sum_{n} x_{n} x_{n-i} \quad \text { for } v \leq i \leq N
$$

Equations such as (15) are called normal equations. They can be solved for the predictor coefficients $\left(b_{j}, v \leq i \leq N\right)$ using $N-v+1$ equations. It is now time to deal with the summation over $n$. There are two possibilities: the autocorrelation method and the covariance method.

\section{AUTOCORRELATION METHOD}

In this case, the summation over $\mathrm{n}$ is taken from $-\infty$ to $\infty$. Equation (15) reduces to

$$
\sum_{j=v}^{N} b_{j} R(i-j)=R(i) \quad \text { for } v \leq i \leq N .
$$

These are known as the Yule-Walker equations. ${ }^{1} \mathrm{R}(\mathrm{k})$ is called the short-time autocorrelation function and is defined by

$$
R(k)=\sum_{n=-\infty}^{\infty} x_{n} x_{n+k}
$$

$R(i)$ is an even function of $i$. That is,

$$
R(-i)=R(i) .
$$

The coefficients of $R(i-j)$ form what is called an autocorrelation matrix. The main difficulty with the implementation of Eq. (17) is that $x_{n}$ is usually not known over $-\infty$ to $\infty$. It is usually only known over some interval

$$
0 \leq n \leq M-1 .
$$

The method used to deal with this problem is windowing. A window is defined over $0 \leq n \leq M-1$ and is zero outside these bounds in order to correspond to the known data. From Eq. (8) it is seen that the error will be large at the beginning of this interval since these values are trying to be predicted from a signal that is zero. Also the error will be large at the end of this interval since very small values are being predicted from much larger (less windowed) values. For these reasons a window with tapered ends is used (usually a Hamming window). The interval in Eq. (19) corresponds to the known data sequence as follows,

$$
x_{n-M}, \ldots, x_{n-2}, x_{n-1} \quad \text { used to predict } x_{n} .
$$

The window is centered over $x(n-M / 2)$. This tends to emphasize the points around the middle of the known data (not the most recent information). As will be seen, this is fine for deterministic or wide-sense stationary data sequences. $R(k)$ is now calculated using Eq. (21).

$$
R(k)=\sum_{m=0}^{M-1-k}\left[x_{n-1-m} h_{m}\right]\left[x_{n-1-m-k} h_{m-k}\right]
$$

where $h_{n}$ is the window defined by 


$$
h_{n}=\left\{\begin{array}{rr}
0.54-0.46 \cos \left(\frac{2 \pi n}{M}\right), & 0 \leq n \leq M-1 \\
0, & \text { otherwise }
\end{array}\right.
$$

The autocorrelation matrix formed by Eq. (16) is a symmetric Toeplitz matrix. A Toeplitz matrix has equal components along each diagonal. A procedure known as Durbin's recursive procedure works very efficiently to solve this system. ${ }^{2}$ Other techniques are also possible. Durbin's procedure is given below.

$$
E_{0}=R(0)
$$

$$
k_{i}=\frac{R(i)+\sum_{j=1}^{i-1} b_{j}^{(i-1)} R(i-j)}{E_{i-1}} .
$$

$$
b_{i}^{(i)}=k_{i} .
$$

$$
\begin{aligned}
& b_{j}^{(i)}=b_{j}^{(i-1)}+k_{i} b_{i-j}^{(i-1)} \quad \text { for } 1 \leq j \leq i-1 . \\
& E_{i}=\left(1-k_{i}^{2}\right) E_{i-1} .
\end{aligned}
$$

Solve Eqs. (24) through (27) iteratively for $\mathrm{i}=1,2, \ldots, \mathrm{N}$. The final predictor coefficients are given by

$$
b_{j}=b_{j}^{(N)}, \quad 1 \leq j \leq N .
$$

$\mathrm{E}_{\mathrm{i}}$ is the minimum total squared error for i filter weights. It can also be computed from,

$$
E_{i}=R(0)-\sum_{j=v}^{N} b_{j} R(j)
$$

This can be used to help find the order of the optimal predictor.

Below are the Yule-Walker equations in matrix form (for $v=1$ ).

$$
\left[\begin{array}{ccccc}
R(0) & R(1) & R(2) & \ldots & R(N-1) \\
R(1) & R(0) & R(1) & \ldots & R(N-2) \\
R(2) & R(1) & R(0) & \ldots & R(N-3) \\
\vdots & \vdots & \vdots & \ddots & \vdots \\
R(N-1) & R(N-2) & R(N-3) & \ldots & R(0)
\end{array}\right]\left[\begin{array}{c}
b_{1} \\
b_{2} \\
b_{3} \\
\vdots \\
b_{N}
\end{array}\right]=\left[\begin{array}{c}
R(1) \\
R(2) \\
R(3) \\
\vdots \\
R(N)
\end{array}\right] .
$$




\section{COVARIANCE METHOD}

In this case, the summation over $n$ is taken over the finite interval $0 \leq n \leq M-1$. Equation (15) reduces to

$$
\sum_{j=v}^{N} b_{j} \phi(i, j)=\phi(i, 0) \quad \text { for } v \leq i \leq N
$$

where

$$
\phi(i, j)=\sum_{m=0}^{M-1} x_{m-i} x_{m-j}, \quad\left\{\begin{array}{l}
1 \leq i \leq N \\
0 \leq j \leq N .
\end{array}\right.
$$

This requires values of $x_{n}$ from $-N \leq n \leq M-1$ (N+M total samples). The data is not windowed as in the autocorrelation method since data is required from outside the $0 \leq n \leq \mathrm{M}-1$ interval. The function $\phi$ in Eq. (32) is not a true autocorrelation function, but a cross-correlation between similar (but not identical) data sequences. Note that the covariance method is similar to the autocorrelation method as the summation over $n$ goes to infinity.

An example of the normal equations for the covariance method is given in Eq. (33) from Eq. (31) for $v=1$.

$$
\left[\begin{array}{ccccc}
\phi(1,1) & \phi(1,2) & \phi(1,3) & \ldots & \phi(1, N) \\
\phi(2,1) & \phi(2,2) & \phi(2,3) & \ldots & \phi(2, N) \\
\phi(3,1) & \phi(3,2) & \phi(3,3) & \ldots & \phi(3, N) \\
\vdots & \vdots & \vdots & \ddots & \vdots \\
\phi(N, 1) & \phi(N, 2) & \phi(N, 3) & \ldots & \phi(N, N)
\end{array}\right]\left[\begin{array}{c}
b_{1} \\
b_{2} \\
b_{3} \\
\vdots \\
b_{N}
\end{array}\right]=\left[\begin{array}{c}
\phi(1,0) \\
\phi(2,0) \\
\phi(3,0) \\
\vdots \\
\phi(N, 0)
\end{array}\right]
$$

This matrix has the properties of a covariance matrix and hence the name of the method. From Eq. (32), $\phi(\mathrm{i}, \mathrm{j})=\phi(\mathrm{j}, \mathrm{i})$, and so the square matrix in Eq. (33) is symmetric, but is not Toeplitz. This can be seen from Eq. (32),

$$
\phi(i+1, j+1)=\phi(i, j)+x_{-i-1} x_{-j-1}-x_{M-i-1} x_{M-j-1} .
$$

A technique called Cholesky decomposition ${ }^{3,4}$ or a technique called LU (Lower Upper) decomposition ${ }^{4}$ can be used efficiently here. The error $E_{i}$ is

$$
E_{i}=\phi(0,0)-\sum_{j=v}^{N} b_{j} \phi(j, 0)
$$

Now assume that $x_{n}$ is a sample function of a random process. Equation (9) becomes

$$
E_{v}=E\left[\varepsilon_{n, v}^{2}\right]=E\left[\left(x_{n}-\sum_{j=v}^{N} b_{j} x_{n-j}\right)^{2}\right]
$$


and the normal equations become

$$
\sum_{j=v}^{N} b_{j} E\left[x_{n-j} x_{n-i}\right]=E\left[x_{n} x_{n-i}\right], \quad v \leq i \leq N
$$

where $\mathrm{E}[]$ is the ensemble average. The taking of this average depends on the degree of stationarity of the random process.

If $\mathrm{x}_{\mathrm{n}}$ comes from a wide-sense stationary process, it is also assumed that the process is ergodic. This might not always be true, but must be done in order to get a solution. Since the simulation results were found acceptable, this assumption is validated.

If the process is ergodic, the ensemble average is equivalent to a time average and

$$
E\left[x_{n-j} x_{n-i}\right]=R(i-j)
$$

and

$$
E\left[x_{n} x_{n-i}\right]=R(i) .
$$

Equation (37) is now identical to Eq. (16) and thus the autocorrelation method applies to the wide-sense stationary case. Note also that in the wide-sense stationary ergodic case the covariance method reduces to the Wiener or optimal solution. ${ }^{5}$ Thus the covariance method also works for wide-sense stationary processes. becomes

If $x_{n}$ comes from a nonstationary random process, then the ensemble average from Eq. (37)

$$
E\left[x_{n-j} x_{n-i}\right]=R(n-j, n-i)
$$

where $R(i, j)$ is the nonstationary autocorrelation between samples at times $i$ and $j$. This is now a function of $n$. Since the random process is obviously not ergodic, the time average cannot be used for $\mathrm{E}[]$ in Eq. (40). Since only one sample function, $x_{n}$, from the random process is known, the ensemble average in Eq. (40) cannot be computed. Hence the nonstationary case cannot be solved in this manner. Generally, iterative techniques such as the LMS algorithm ${ }^{5}$ or the Kalman filter ${ }^{1}$ are used. For now it should be stated that if the random process can be considered locally stationary (i.e., stationary for short segments of time), it is possible to estimate the autocorrelation function (in Eq.

(40)) using a short-time average. Use

$$
R(-j,-i) \approx \phi(i, j) .
$$

This leads to the same solution given by the covariance method. Proper choices of $\mathrm{N}$ and $\mathrm{M}$ are necessary in order to deal with the nonstationary transition regions. Note that if the random process is stationary, then $R(i, j)=R(j, i)$ and the system can be solved by the autocorrelation method.

Further information on digital signal processing and linear prediction techniques can be found in the general references. ${ }^{6,7}$

\section{RESULTS AND CONCLUSIONS}

The data used for the simulation were gathered at Argonne National Laboratory in the fall of 1988. The accelerator was part of the test stand for the LANL GTA telescope designed and built by the Accelerator Technology (AT) division. The beam of $\mathrm{H}^{-}$particles had an average beam current of $1.7 \mathrm{~mA}$. The beam repetition rate was $3 \mathrm{~Hz}$. Before sampling, the data was filtered using a 9 th-order 
Tchebyshev low-pass filter with a cutoff frequency of $600 \mathrm{kHz}$. There were 185 useful data points per macropulse.

The simulation used a series of five macropulses numbered $x 1, x 2, x 3, x 4, x 5$, and $y 1, y 2, y 3$, $y 4, y 5$. All jitter data was in the form of beam position versus time. Note that the ability to apply position correction directly implies the ability to apply beam trajectory-angle correction with the correct change in hardware. Twenty-one filter weights were used for both the autocorrelation and covariance methods. The estimated setup for a real control system required prediction two points into the future $(v=2)$. Three sets of runs were performed for each data set ( $x$ and $y)$ for each method. Run \#1 contains data for the model formed using macropulse number 1 ( $x 1$ or $y 1)$. The model is then used to predict the remaining macropulses ( 2 through 5 ) and an rms error is calculated for each. Run \#2 uses an ensemble-average of pulses 1 through 4 to produce a model to predict macropulse number 5. Run \#3 includes the model formed using macropulse number 4 for the prediction of number 5 . This was done to facilitate comparison with run \#2.

The predictive feedforward techniques are compared to a negative feedback control system. A standard one-loop closed negative feedback system was used. The Nyquist stability criterion was used to ensure stability. The details of the feedback system will not be given.

The analysis results are given in Table I. The values for $R_{\text {orig }}$ are the $r m s$ jitter reduction amounts over those with no correction applied (given in $\mathrm{dB}$ ).

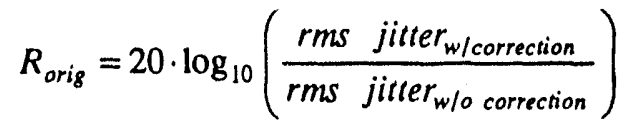

The values for $R_{\text {feed }}$ are the rms jitter reduction amounts over those for the standard feedback system (also given in $\mathrm{dB}$ ).

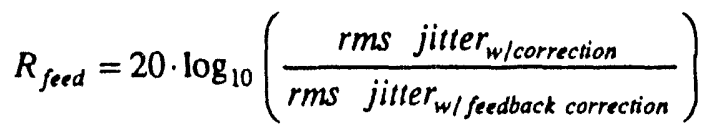

\section{TABLE I}

\section{AUTOCORRELATION, COVARIANCE, AND STANDARD FEEDBACK JTTER REDUCTION}

\begin{tabular}{rcccccr} 
& \multicolumn{2}{c}{ Standard Feedback } & \multicolumn{2}{c}{ Autocorrelation } & \multicolumn{2}{c}{ Covariance } \\
Data & $\mathrm{R}_{\text {orig }}(\mathrm{dB})$ & $\mathrm{R}_{\text {feod }}(\mathrm{dB})$ & $\mathrm{R}_{\text {orig }}(\mathrm{dB})$ & $\mathrm{R}_{\text {feed }}(\mathrm{dB})$ & $\mathrm{R}_{\text {orig }}(\mathrm{dB})$ & $\mathrm{R}_{\text {feed }}(\mathrm{dB})$ \\
Run \#1 x2 & -13.5459 & 0.0000 & -20.1448 & -6.5988 & -20.3536 & -6.8076 \\
×3 & -13.9681 & 0.0000 & -19.4756 & -5.5077 & -19.5051 & -5.5371 \\
x4 & -14.2699 & 0.0000 & -19.7701 & -5.5001 & -19.8060 & -5.5360 \\
×5 & -14.3075 & 0.0000 & -20.2580 & -5.9510 & -20.3559 & -6.0489 \\
Run \#2 x5 & -14.3075 & 0.0000 & -20.1509 & -5.8439 & -20.3168 & -6.0098 \\
Run \#3 x5 & -14.3075 & 0.0000 & -20.0336 & -5.7266 & -20.3062 & -5.9992 \\
Run \#1 y2 & -2.5808 & 0.0000 & -7.1685 & -4.5875 & -7.2732 & -4.6922 \\
y3 & -2.4246 & 0.0000 & -8.4391 & -6.0141 & -8.6879 & -6.2629 \\
y4 & -2.6555 & 0.0000 & -8.6295 & -5.9745 & -8.8076 & -6.1526 \\
y5 & -2.2967 & 0.0000 & -8.4319 & -6.1349 & -8.5227 & -6.2257 \\
Run \#2 y5 & -2.2967 & 0.0000 & -8.5722 & -6.2752 & -8.6033 & -6.3063 \\
Run \#3 y5 & -2.2967 & 0.0000 & -8.3705 & -6.0735 & -8.4424 & -6.1454
\end{tabular}

Both the autocorrelation and covariance methods worked about a factor of two ( $-6 \mathrm{~dB})$ better than the standard feedback method. This trend held for almost all of the cases. The covariance method worked slightly better than the autocorrelation method, but both techniques gave very comparable results. The feedback system had great difficulties with the $y$-plane data. The 
feedforward systems also displayed some difficulties with this data, but still outperformed the feedback system with a margin equal to that of the $x$-plane data. This was due to the lack of richness of deterministic frequency components in the $y$-plane data. The predictions for point 5 (both $x$ and $y$ ) seemed to be about equal for the different training macropulses used. This would seem to indicate that the data was stationary over the time observed. The data is at least locally wide-sense stationary on a pulse-to-pulse basis.

An analysis of the correction bandwidth for the feedforward systems showed a vast improvement over that of the feedback system. The average correction bandwidth for the feedback system was $50 \mathrm{KHz}$ compared to roughly $600 \mathrm{KHz}$ for the feedforward systems. This is quite understandable when one compares the virtues of feedforward control to those of feedback control. The advantage of feedforward control is that of larger correction bandwidth. What is lost is absolute stability thus requiring an extra BPM and the complexity of its processing requirements. The feedback system is much simpler to implement, but if improved jitter correction and bandwidth are desired, the complexity and cost of a predictive system may be necessary.

\section{REFERENCES}

1. S. Haykin, Adaptive Filter Theory, (Prentice-Hall, Inc., Englewood Cliffs, N.J., 1986).

2. J. Makhoul, IEEE Trans. on ASSP, 25(5), 423 (1977).

3. J.J. Dongarra, C.B. Moler, J.R. Bunch, G.W. Stewart, Linpack Users' Guide, (Siam, Philadelphia, Penn., 1979).

4. L.R. Rabiner, R.W. Schafer, Digital Signal Processing of Speech Signals, (Prentice-Hall, Inc., Englewood Cliffs, N.J., 1978).

5. B. Widrow, S. Stearns, Adaptive Signal Processing, (Prentice-Hall, Inc., Englewood Cliffs, N.J., 1985).

6. A.V. Oppenheim, R.W. Schafer, Discrete-Time Signal Processing, (Prentice-Hall, Inc., Englewood Cliffs, N.J., 1989).

7. J.L. Doob, Stochastic Processes, (John Wiley and Sons, Inc., N.Y., 1953).

\section{DISCLAIMER}

This report was prepared as an account of work sponsored by an agency of the United States Government. Neither the United States Government nor any agency thereof, nor any of their employees, makes any warranty, express or implied, or assumes any legal liability or responsibility for the accuracy, completeness, or usefulness of any information, apparatus, product, or process disclosed, or represents that its use would not infringe privately owned rights. Reference herein to any specific commercial product, process, or service by trade name, trademark, manufacturer, or otherwise does not necessarily constitute or imply its endorsement, recommendation, or favoring by the United States Government or any agency thereof. The views and opinions of authors expressed herein do not necessarily state or reflect those of the United States Government or any agency thereof. 


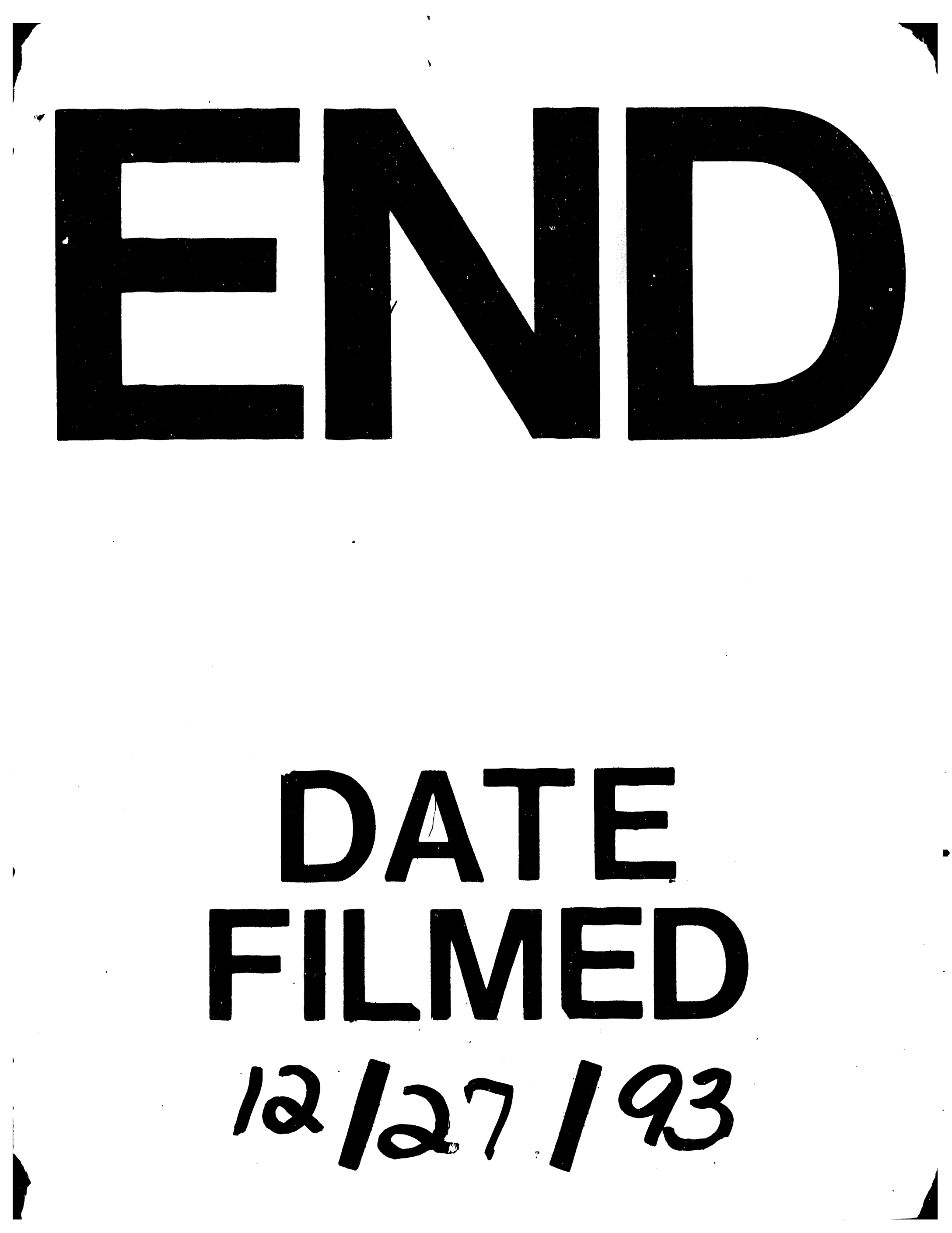


